\title{
Suppression of Decomposition of Propylene Carbonate at Graphite Electrodes by the Addition of Nonionic Surfactants
}

\author{
Akira KITANI, * Tsukasa FUKUTA, Yorimitsu KODAMA, \\ Nobuaki OHTA, and Sotaro ITO
}

\begin{abstract}
Department of Chemistry and Chemical Engineering, Graduate School of Engineering, Hiroshima University (1-4-1 Kagamiyama, Higashi-Hiroshima 739-8527, Japan)
\end{abstract}

Received May 29, 2003 ; Accepted August 5, 2003

\begin{abstract}
The effects of nonionic surfactants on electrochemical behaviors of graphite electrodes in propylene carbonate solutions containing $\mathrm{Li}$ ion were studied with a cyclic voltammetry. The reductive decomposition of propylene carbonate was markedly suppressed by the addition of nonionic surfactants such as polyethylene glycol monolaurate or polyethylene glycol monomethyl ether, and the anodic peak due to the deintercalation of $\mathrm{Li}$ ion from graphite electrode was clearly observed in the presence of surfactants. The addition of such kind of surfactants is highly useful for the development of Li ion secondary battery utilizing propylene carbonate.
\end{abstract}

Key Words : Propylene Carbonate, Graphite Electrode, Nonionic Surfactant, Additive Effect

\section{Introduction}

The suppression of the reductive decomposition of propylene carbonate (PC) at graphite electrodes is an important problem for the development of high performance lithium ion batteries. The addition of crown ether or catechol carbonate to PC solutions is known to be effective to suppress the solvent decomposition. ${ }^{1,2)}$ However, the development of more effective suppressor is needed.

On the other hand, we are studying the effect of surfactants on organic electrode reactions. We have found that products distribution could be controlled by using cationic surfactants in the cathodic reduction of acetophenone. ${ }^{3-5)}$ Surfactants may affect reaction pathways of various types of organic electrode reactions.

In this research, polyethylene glycol type nonionic surfactants, which are expected to interact with $\mathrm{Li}$ cation, were added in PC solutions containing $\mathrm{Li}$ ion to suppress the decomposition of the solvent.

\section{Experimental}

The nonionic surfactants were commercially available and used without further purification. Two types of surfactants were used, the one was polyethylene glycol monolaurate $\left[\mathrm{C}_{11} \mathrm{H}_{23} \mathrm{CO}\left(\mathrm{OC}_{2} \mathrm{H}_{4}\right)_{\mathrm{n}} \mathrm{OH}, \quad \mathrm{n}=10\right]$ and the other was polyethylene glycol monomethyl ether $\left[\mathrm{CH}_{3}\left(\mathrm{OC}_{2} \mathrm{H}_{4}\right)_{\mathrm{n}} \mathrm{OH}, \mathrm{n}=7.2\right]$.

Graphite powder (Nakarai Chemicals) was compressed to form a pellet with adding $20 \%$ Teflon powder. A graphite pellet was used as a working and a Pt plate was used as a counter electrode. Silver wire was used as a quasi-reference electrode $(\mathrm{E}(\mathrm{Ag})=-0.19 \mathrm{~V}$ vs Ferrocene $/$ Ferricinium, $\mathrm{Fc} / \mathrm{Fc}^{+}$) and $\mathrm{LiClO}_{4}$ was used as a supporting electrolyte.

Sample electrolyte solutions were prepared using a vacuum line to avoid the moisture and oxygen. Molecu- lar sieve $3 \mathrm{~A}$ or $\mathrm{P}_{2} \mathrm{O}_{5}$ was used as a drying agent. Electrochemical behaviors of graphite electrodes in PC (Kishida Chemicals) solutions were studied with a cyclic voltammetry under nitrogen atmosphere.

\section{Results and Discussion}

Figure 1 shows cyclic voltammograms at graphite electrodes in PC solutions containing $0.1 \mathrm{M}\left(\mathrm{M}=\mathrm{mol} \mathrm{dm}^{-3}\right)$ $\mathrm{LiClO}_{4}$. An anodic peak due to the deintercalation of $\mathrm{Li}$ ion was not observed in the absence of surfactant (Fig. 1a). In this case, most of cathodic charge was consumed for the decomposition of the solvent.

In the presence of $0.02 \mathrm{M}$ polyethylene glycol monolaurate, cathodic currents were decreased and an anodic peak due to the deintercalation of $\mathrm{Li}$ ion from graphite electrode was clearly observed (Fig. 1b). The cathodic charge $\left(Q_{c}\right)$, determined form the integration of voltammogram, became almost same as the anodic charge $\left(Q_{a}\right)$ at the second cycle. This behavior indicates reversible intercalation-deintercalation reaction of $\mathrm{Li}$ ion took place without accompanying the decomposition of PC.

The effect of surfactant concentration on the voltammetric behavior (second cycle) of graphite electrode is shown in Fig. 2. In this figure, $Q_{a}$ and $Q_{c}-Q_{a}$, difference between $Q_{c}$ and $Q_{a}$, are shown as the measure of reversible and irreversible capacity of graphite electrode. The optimum concentration of polyethylene glycol monolaurate added was determined to be $0.02 \mathrm{M}$. At higher surfactant concentration of $0.05 \mathrm{M}$, both cathodic and anodic currents markedly decreased. Maybe thick layer of nonconductive adsorbed surfactant inhibits all kinds of electrode reactions.

In order to examine the effect of long alkyl chain of surfactant, polyethylene glycol monomethyl ether was added to PC solution instead of monolaurate (Fig. 3). Al- 


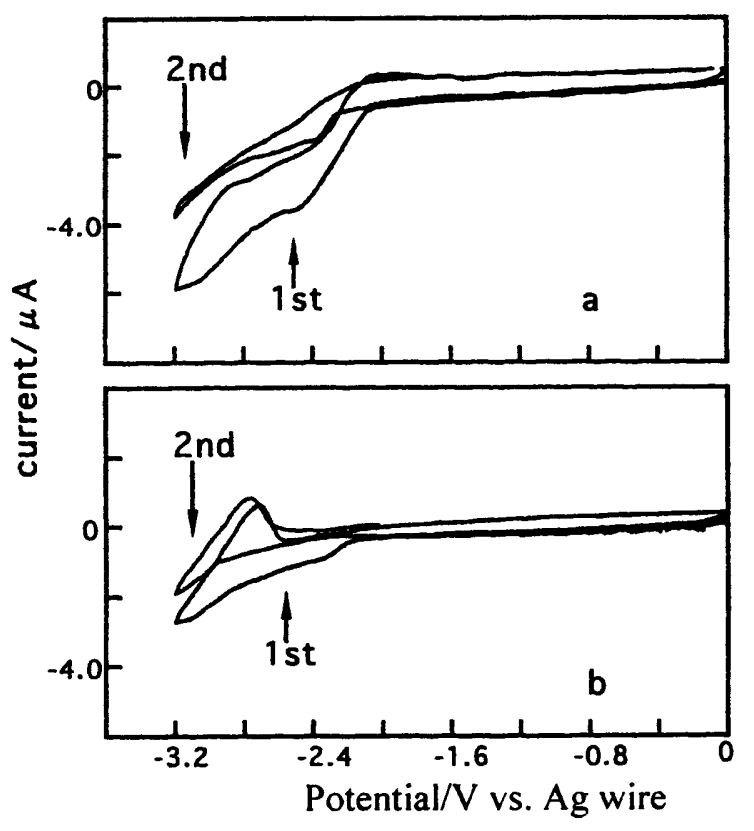

Fig. 1 Effect of polyethylene glycol monolaurate on voltammetric behaviors of graphite electrodes in $0.1 \mathrm{M} \mathrm{LiClO}_{4} / \mathrm{PC}$ solution at $1 \mathrm{mV} / \mathrm{s}$. Attached symbols (1st and 2nd) indicate the first and second cycle, respectively. a : without surfactant, b : $0.02 \mathrm{M}$ surfactant added.

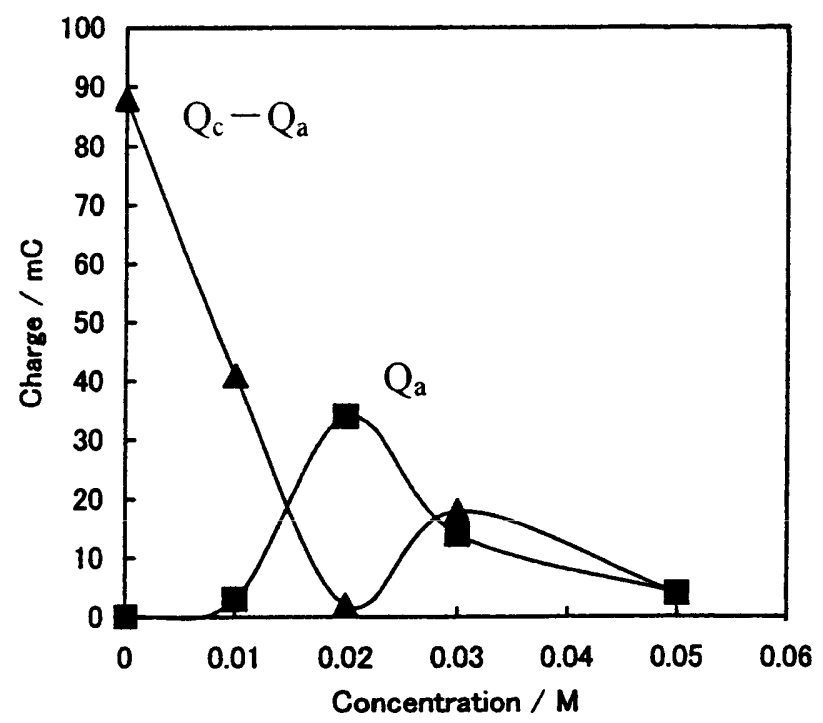

Fig. 2 Effect of surfactant concentration on voltammetric behavior (2nd cycle) of graphite electrode. Square : $\mathrm{Q}_{\mathrm{a}}$, Triangle : $\mathrm{Q}_{\mathrm{c}}-\mathrm{Q}_{\mathrm{a}}$.

though the suppression of solvent decomposition at the first cycle was not so remarkable, a well defined anodic peak was observed in the reverse scan. At the second cycle, almost same voltammogram was obtained as that shown in Fig. 1b. Therefore, polyethylene glycol moiety contained in surfactants plays an important role for the suppression of decomposition of PC.

The marked effect of polyethylene glycol type surfactants on electrochemical behaviors of graphite electrodes

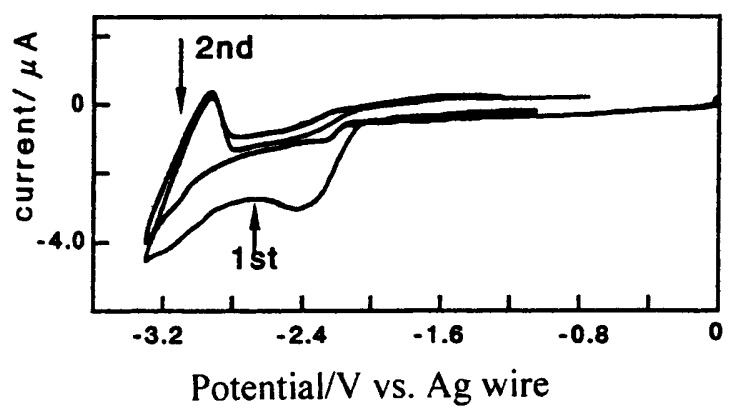

Fig. 3 Cyclic voltammogram obtained in the presence of $0.02 \mathrm{M}$ polyethylene glycol monomethyl ether. Other conditions are same as those described in Fig. 1.

may be due to the adsorption of surfactants onto graphite electrode surface. The properly adsorbed layer may act as an SEI (Solid Electrolyte Interface) film formed in propylene carbonate solution, and reversible intercalation-deintercalation reaction of $\mathrm{Li}$ ion at graphite electrode may take place.

The surfactants used in this study are electroinactive up to $-3.2 \mathrm{~V}$, since direct reduction wave of surfactants was not observed with a glassy carbon electrode. For practical use in lithium ion secondary batteries, they should be stable toward anodic oxidation. Voltammetric measurements are made in PC solution with a glassy carbon electrode. In both cases, no oxidation wave of surfactant itself was observed up to $2.0 \mathrm{~V}$.

\section{Conclusion}

This work clearly demonstrates that the addition of small amount of polyethylene glycol type nonionic surfactant is quite useful for the suppression of decomposition of PC. Reversible intercalation-deintercalation reaction of $\mathrm{Li}$ ion is possible without accompanying the solvent decomposition. The surfactants studied should be stable during charging and discharging process of lithium ion secondary batteries. Studies on the suppressing effect of other polyethylene glycol type surfactants with different alkyl group are in progress.

\section{Acknowledgement}

This work is partly supported by the Electric Technology Research Foundation of Chugoku.

\section{References}

1) Z. X. Shu, R. S. McMillan, J. J. Murray, and J. Davidson, J. Electrochem. Soc., 142, L 161 (1995).

2) C. Wang, H. Nakamura, H. Komatsu, M. Yoshio, and H. Yoshitake, J. Power Sources, 142, 74 (1998).

3) Y. Kodama, M. Imoto, N. Ohta, A. Kitani, and S. Ito, Chem. Lett., 1997, 337.

4) Y. Kodama, A. Fujiwara, H. Kawamoto, N. Ohta, A. Kitani, and S. Ito, Chem. Lett., 2001, 240.

5) Y. Kodama, M. Imoto, N. Ohta, A. Kitani, and S. Ito, J. Electroanal. Chem., 507, 103 (2001). 